\title{
Radiographic diagnosis of a rare case of oculodentodigital dysplasia
}

\author{
Umesh Chandra Parashari, M.D. \\ Sachin Khanduri, M.D. \\ Samarjit Bhadury, M.D. \\ Fareena Akbar Qayyum, M.B.B.S. \\ Department of Radiodiagnosis, Lucknow Medical College, Lucknow, Uttar Pradesh, India
}

Corresponding author: U Parashari (drumesh.rd@gmail.com)

\begin{abstract}
Oculodentodigital dysplasia (ODDD), also known as oculodentoosseous dysplasia, is an extremely rare autosomal dominant disorder with high penetrance, intra- and interfamilial phenotypic variability, and advanced paternal age in sporadic cases. The incidence of this disease is not precisely known, with only 243 cases reported in the scientific literature, suggesting an incidence of around 1 in 10 million people. It is marked mainly by eye abnormalities, craniofacial dysmorphism, dental anomalies, hand and foot malformations, various skeletal defects, and mildly delayed mental development. Neurological changes may appear earlier in each subsequent generation. This case report describes a radiological diagnosis of ODDD based on physical appearance, clinical features and radiographic findings in a 16-year-old girl.
\end{abstract}

\section{Introduction}

Oculodentodigital dysplasia (ODDD) is a condition that affects many parts of the body, particularly the eyes, teeth and fingers, as the term indicates. Mutations in the connexin 43 gene or gap junction protein alpha-1 gene (GJA1) located on chromosome 6q22-q24 lead to disruption of $\mathrm{Cx} 43$-mediated cell-to-cell communication, resulting in disrupted morphological patterning during development and altered functioning of cells in mature tissue. Most cases of ODDD are inherited in an autosomal dominant pattern, which means one copy of the altered gene in each cell is sufficient to cause the disorder. Less commonly, ODDD can be inherited in an autosomal recessive pattern, which means both copies of the gene in each cell have mutations. The condition is also known as oculodento-osseous syndrome or Meyer-Schwickerath syndrome. Craniofacial abnormalities include a thin nose with hypoplastic alae nasi, small anteverted nares, prominent columnella, and microcephaly. Brittle nails and hair abnormalities of hypotrichosis and slow growth are also prominent. Common ocular features with this condition are microphthalmia, microcornea, fine porous spongy iris abnormalities, cataracts, glaucoma, optic atrophy and other eye abnormalities that can lead to vision loss. Abnormalities seen in the orodental region are small or missing teeth, weak enamel, multiple cavities and early tooth loss. Other common features of this condition include a thin nose and webbing of the skin (syndactyly) between the fourth and fifth fingers. There is also evidence of camptodactyly and clinodactyly owing to hypoplasia or aplasia of the middle phalanges. siobhCranial hyperostosis and broad tubular bones may be present.

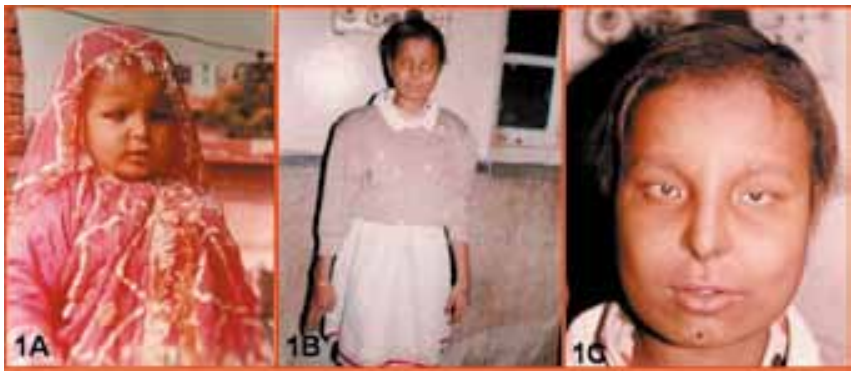

Fig. 1. Photograph of the patient at age one year (1A) and 16 years (1B and 1C) showing hypotrichosis and pili annulati. The face is small with narrow eyes, thin nose, prominent columella and wide mandible. The fingers are underdeveloped and deformed.

\section{Case report}

A 16-year-old girl presented to the hospital with complaints of weakness in her lower limbs, abnormal dentition and bladder incontinence. On general examination, her gait was ataxic with moderate spasticity in both legs. She had dry and scaly skin and brittle nails. Hair growth was sparse and showed characteristics of hypotrichosis and pili annulati. Examination of the face revealed small and narrow eyes with thin and sparse eyebrows. She also had a thin nose with prominent columella and wide lower jawbone. On skeletal examination, her hands revealed underdeveloped fingers and toes with camptodactyly of the 5th (little) fingers on both sides, and syndyctaly of the thumbs and $2 \mathrm{nd}$, 3rd and 4 th fingers. The feet revealed syndyctaly of the 2 nd to 5 th toes and clinodactyly of the great toes. Oral cavity examination revealed localised arrested tooth development that mainly involved the anterior teeth on one side of the midline, mainly the maxillary central and lateral incisors. Radiographically, the teeth had a ghost-like appearance. Calcification and bits of prismatic enamel were found in the pulp, and the enamel was thin and absent in parts. Various X-rays of both the limbs were taken, revealing broad tubular bones and marked hypoplasia of the middle phalanges in the 5th fingers and in all toes. The cranial bones were hyperostotic, and the long bones as well as the ribs and clavicles showed widening. On CT of the face, the rami of the mandible appeared bulky and showed features of mandibular retrognathism. CT of the head also revealed bilateral basal ganglia calcification. The patient did not have any features of mental retardation. On ophthalmic examination, she was diagnosed with cataract, diminished visual acuity and reduced tear secretion. She also had small corneas and eccentric pupils. Otoscopy revealed conductive hearing loss in the right ear. After summing up 


\section{CASE REPORT}

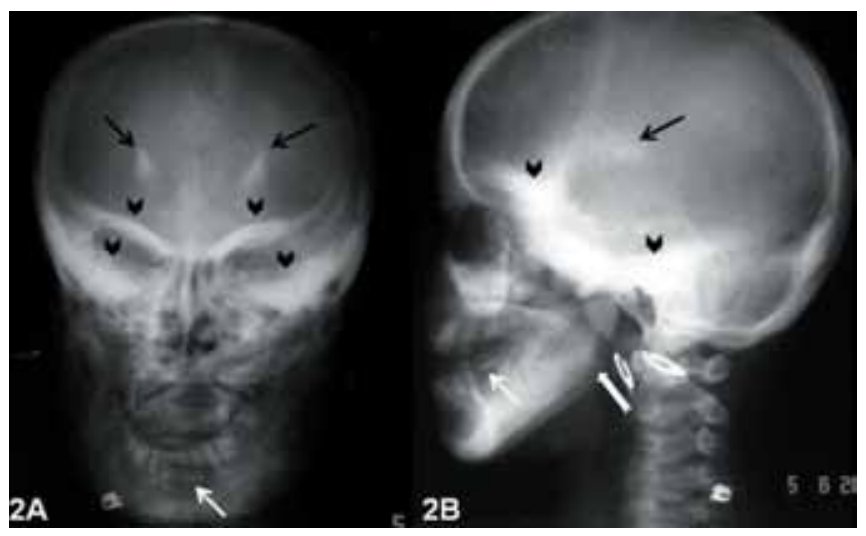

Fig. 2. AP X-ray of the skull (2A) and lateral (2B) view show a ghost-like appearance ('alien look') with cranial hyperostosis (black arrowheads). Focal bilateral symmetrical intracranial calcification is seen (black arrows). Thinned out and focally absent enamel with missing teeth and cavities are seen (thin white arrows) with broadened mandible (thick white arrow).

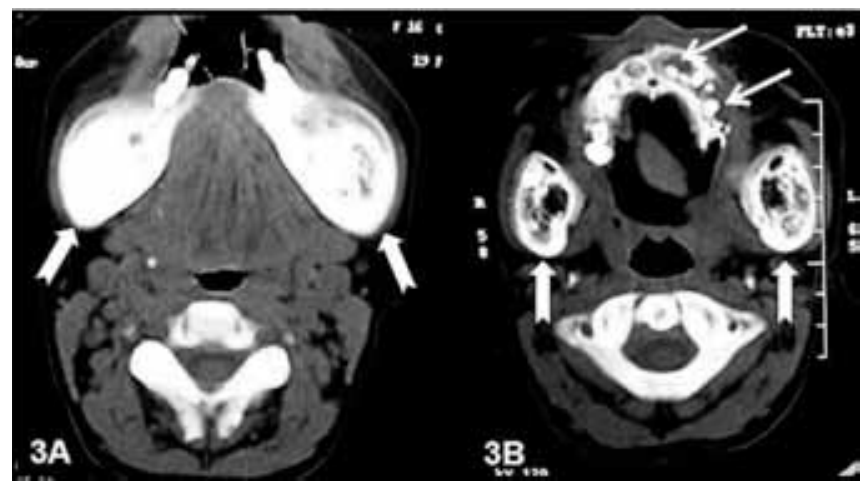

Fig. 3. CT of the face ( $3 A$ and $3 B)$ show bulky rami of the mandible with mandibular retrognathism (thick white arrows). Microdontia, partial anodontia, enamel hypoplasia, multiple caries and early tooth loss are seen (thin white arrows).

all the clinical features, various clinical examinations and radiological evaluations, the diagnosis of ODDD was made.

\section{Discussion}

ODDD is a rare autosomal dominant congenital disorder mainly affecting the development of the face, eyes, skeletal system, heart and dentition. ODDD has been mapped to chromosome 6q22-q24 and germline mutations have been identified in the connexin 43 gene, GJA1. To date, over 70 reports in the literature describe the clinical features of ODDD in more than 240 patients, the majority of whom were white. Typical craniofacial anomalies include a thin nose with hypoplastic alae nasi, small anteverted nares, prominent columnella, and microcephaly. Brittle nails and hair abnormalities of hypotrichosis and slow growth are present. ${ }^{1}$ Some cases have dysplastic ears and conductive hearing loss. Ophthalmic findings include microphthalmia, microcornea, fine porous spongy iris abnormalities, cataracts, glaucoma and optic atrophy. Anomalies observed in the oral region are mandibular overgrowth and cleft palate. The majority of cases have abnormal primary and permanent dentition with microdontia, partial anodontia, enamel hypoplasia, numerous caries, and early tooth loss. Hand and foot abnormalities in ODDD include syndactyly involving the 3rd, 4th and 5th fingers and 2nd to 4th toes, camptodactyly, and clinodactyly owing to hypoplasia or aplasia of the middle phalanges. ${ }^{2}$ Other skeletal

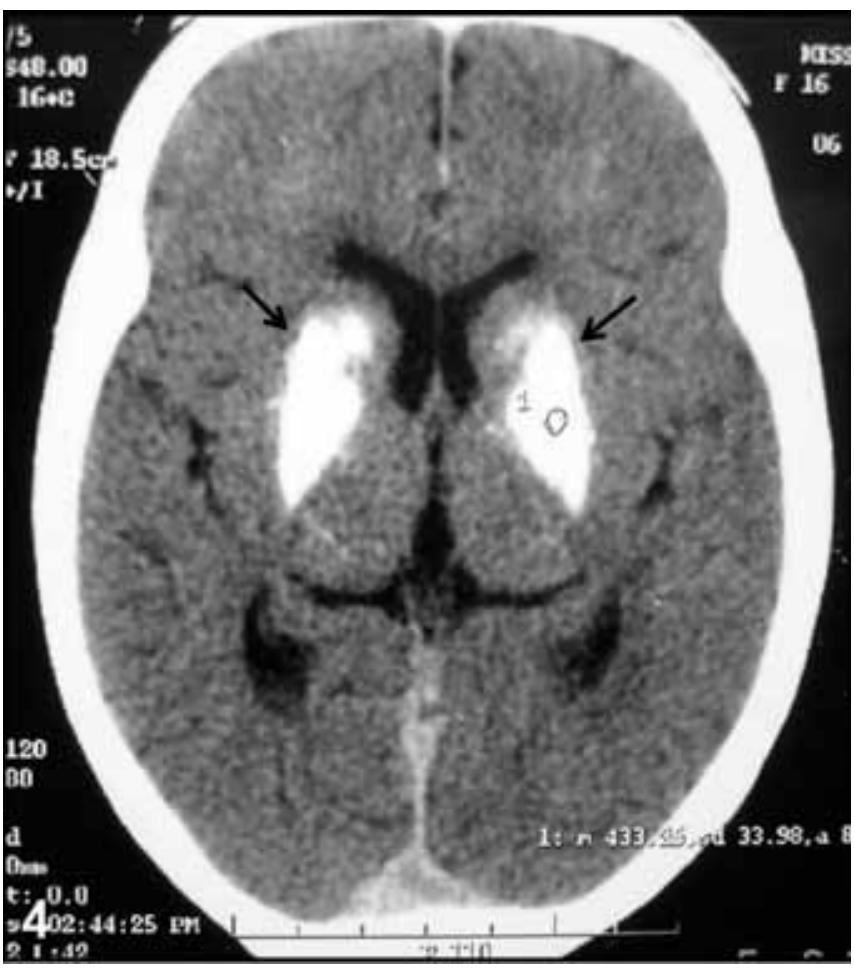

Fig. 4. Non-contrast CT of the head shows dense bilateral basal ganglia calcification (black arrows).

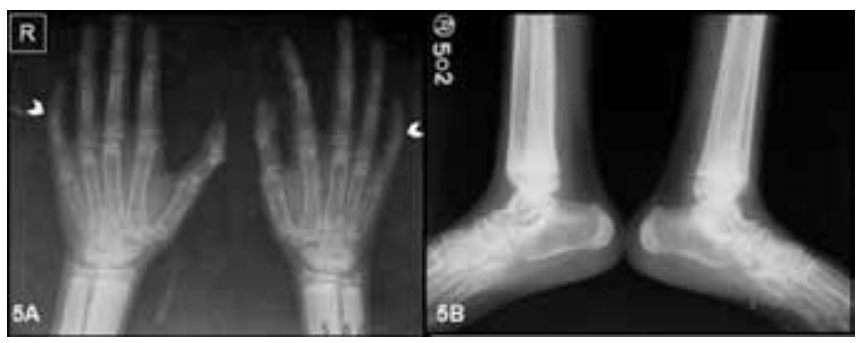

Fig. 5. X-rays of both hands with AP view (5A) and ankles with lower leg lateral view (5B) reveal broad tubular bones and marked hypoplasia of the middle phalanges in the fifth fingers (white arrowheads) with camptodactyly.

abnormalities are cranial hyperostosis, mandible with wide alveolar ridge, and broad tubular bones. Neurological symptoms are inconsistent but frequent, and include dysarthria, neurogenic bladder disturbances, spastic paraparesis, ataxia, anterior tibial muscle weakness, and seizures. ${ }^{3}$ Some patients have dysplastic ears and conductive hearing loss. ODDD is caused by heterozygous mutations in the GJA1 gene (6q22-q23), which encodes the gap junction protein connexin 43 (Cx43). Over 40 causative mutations have been identified. In most cases, ODDD is inherited in an autosomal dominant manner with high penetrance and variable expression. ${ }^{4}$ Advanced paternal age has been noted in sporadic ODDD cases. Diagnosis is based on clinical findings and can be confirmed by molecular studies. Differential diagnoses include a wide number of syndromes that present with skeletal, ocular, dental and neurological manifestations. Genetic counseling should be offered to all ODDD patients. ${ }^{1}$

There are many conditions mentioned in the literature with overlapping features as in ODDD.These include: EEC syndrome, Hallermann-Streiff syndrome, orofacial digital syndrome Type II, KID syndrome etc. The EEC syndrome is characterised by ectrodactyly or 


\section{CASE REPORT}

lobsterclaw deformity, ectodermal dysplasia, and cleft lip and palate. It is a rare disorder with autosomal dominant inheritance, variable expression and, in some families, lack of penetrance. Cleft lip/palate is present in most patients; in those without cleft lip/palate, the philtrum or uvula or both are often abnormal. The cleft lip/palate was not present in our patient, while significant eye changes and facial features favouring ODDD were present. ${ }^{5}$

Hallermann-Streiff syndrome (HSS) is a rare disorder characterised primarily by head and face abnormalities, with dental abnormalities also present. The digital changes, such as syndactyly or camptodactyly, as in ODDD, are not present in the former syndrome. ${ }^{6}$ MohrClaussen syndrome, or oro-facial-digital syndrome type II (OFD-II), is transmitted as an autosomal recessive disorder, and is characterised by malformation of the face, oral cavity and digits. Cleft upper lip and cleft palate, lobulated tongue and digital features include clinodactyly, syndactyly, brachydactyly and duplication of the first toe. There are usually no eye manifestations and skin changes as seen in ODDD. ${ }^{7}$ Keratitis-ichthyosis-deafness (KID) syndrome is the most severe cutaneous connexin disorder because of the involvement of several epithelia of ectodermal origin, including skin, appendages, nails, teeth, inner ear and cornea. Most sufferers develop symmetrical, wellcircumscribed hyperkeratotic plaques with underlying erythema on the extremities and face (erythrokeratoderma).

Chronic cheilitis and perléche are common, whereas hair and nail dystrophy, scarring alopecia, dental anomalies and heat intolerance are less frequent. Differential diagnoses for bilateral symmetrical enlargement of the mandibular rami and anterior symphyseal lesions include ameloblastic fibro-odontoma, cherubism, torus, osteosarcoma and chronic osteomyelitis, but associated syndactyly is absent. ${ }^{8}$ Bilateral basal ganglia calcification is also present in Fahr syndrome, but it occurs between the age of 30 and 35 years of age. Important differential diagnoses of basal ganglia calcification of familial nature are Fahr's syndrome (familial idiopathic symmetrical basal ganglia calcification), Cockayne's syndrome, tubero sclerosis, and familial degenerative disorders. Aicardi-Goutières syndrome is an autosomal recessive encephalopathy that causes developmental arrest, basal ganglia calcification and white matter disease in the presence of chronic cerebrospinal fluid lymphocytosis, and a raised level of cerebrospinal fluid interferon-alpha.

Management is multidisciplinary. Regular follow-up should include complete eye examination and neurological, hearing and dental evaluation. As blindness owing to glaucoma can occur, patients at risk should receive anti-glaucoma treatment. Plastic or orthopaedic surgery is indicated for severe limb malformations. Early recognition of the syndrome is of crucial importance in prevention and treatment of the wide variety of clinical manifestations.

1. Kjaer KW, Hansen L, Eiberg H, Leicht P, Opitz JM, Tommerup N. Novel connexin 43 (GJA1) mutation causes oculo-dento-digital dysplasia with curly hair. Am J Med Genet 2004;127A:152-157.

2. Vitiello C, D'Adamo P, Gentile F, Vingolo EM, Gasparini P, Banfi S. A novel GJA1 mutation causes oculodentodigital dysplasia without syndactyly. Am J Med Genet 2005; 133:58-60.

3. Loddenkemper T, Grote K, Evers S, Oelerich M, Stogbauer F. Neurological manifestations of the oculodentodigital dysplasia syndrome. J Neurol 2002;249:584-595.

4. Paznekas WA, Boyadjiev SA, Shapiro RE, et al. Connexin 43 (GJA1) mutations cause the pleiotropic phenotype of oculodentodigital dysplasia. Am J Hum Genet 2003;72:408-418.

5. Mc Nab AA, Potts MJ, Welham RAN. The EEC syndrome and its ocular manifestations. Br J Ophthalmol 1989;73:261-264.

6. Kirzioglu Z, Ceyhan D. Hallermann-Streiff Syndrome: A case report from Turkey. Med Oral Patol Oral Cir Bucal 2009;14 (5):236-238

7. Biswas A, Ghosh JK, Sinha MKR, Basu K, Chatterjee S. Mohr-Claussen syndrome or Orofacial-digital syndrome (OFDS) Type-II. J Pak Med Assoc 2009;59(7):484-486.

8. Neyaz Z, Gadodia A, Gamanagatti S, Mukhopadhyay S. Radiographical approach to jaw lesions. Singapore Med J 2008;49(2):165. 